APS

physics

This is the accepted manuscript made available via CHORUS. The article has been published as:

First observation of rotational structures in $\wedge\{168\}$ Re

D. J. Hartley, R. V. F. Janssens, L. L. Riedinger, M. A. Riley, X. Wang, S. L. Miller, A. D. Ayangeakaa, P. F. Bertone, M. P. Carpenter, C. J. Chiara, P. Chowdhury, U. Garg, G. Gürdal,

S. S. Hota, F. G. Kondev, T. Lauritsen, W. C. Ma, J. Matta, E. A. McCutchan, S. Mukhopadhyay, E. E. Pedicini, J. R. Vanhoy, and S. Zhu Phys. Rev. C 94, 054329 - Published 30 November 2016 DOI: 10.1103/PhysRevC.94.054329 


\title{
First observation of rotational structures in ${ }^{168} \mathbf{R e}$
}

\author{
D. J. Hartley, ${ }^{1}$ R. V. F. Janssens, ${ }^{2}$ L. L. Riedinger,${ }^{3}$ M. A. Riley, ${ }^{4}$ X. Wang, ${ }^{4}, *$ S. L. Miller ${ }^{4}$ A. \\ D. Ayangeakaa $,{ }^{5}, \dagger$ P. F. Bertone ${ }^{2, \ddagger}$ M. P. Carpenter ${ }^{2}$ C. J. Chiara, ${ }^{2,6,7,}$, P. Chowdhury, ${ }^{8}$ U. \\ Garg, ${ }^{5}$ G. Gürdal, ${ }^{7,}$ ฯ S. S. Hota,${ }^{8, * *}$ F. G. Kondev, ${ }^{7}$ T. Lauritsen, ${ }^{2}$ W. C. Ma, ${ }^{9}$ J. Matta, ${ }^{5, \dagger \dagger}$ \\ E. A. McCutchan, ${ }^{2}, \ddagger$ S. Mukhopadhyay, ${ }^{5,} \S \S$ E. E. Pedicini, ${ }^{1}$ J. R. Vanhoy, ${ }^{1}$ and S. Zhu ${ }^{2}$ \\ ${ }^{1}$ Department of Physics, U.S. Naval Academy, Annapolis, Maryland 21402, USA \\ ${ }^{2}$ Physics Division, Argonne National Laboratory, Argonne, Illinois 60439, USA \\ ${ }^{3}$ Department of Physics and Astronomy, University of Tennessee, Knoxville, Tennessee 37996, USA \\ ${ }^{4}$ Department of Physics, Florida State University, Tallahassee, Florida 32306, USA \\ ${ }^{5}$ Department of Physics, University of Notre Dame, Notre Dame, Indiana 46556, USA \\ ${ }^{6}$ Department of Chemistry and Biochemistry, University of Maryland, College Park, Maryland 20742, USA \\ ${ }^{7}$ Nuclear Engineering Division, Argonne National Laboratory, Argonne, Illinois, 60439, USA \\ ${ }^{8}$ Department of Physics, University of Massachusetts Lowell, Lowell, Massachusetts 01854, USA \\ ${ }^{9}$ Department of Physics, Mississippi State University, Mississippi State, Mississippi 39762, USA
}

First rotational sequences have been assigned to the odd-odd nucleus ${ }^{168}$ Re. Coincidence relationships of these structures with rhenium $\mathrm{x}$ rays confirm the isotopic assignment, while arguments based on the $\gamma$-ray multiplicity ( $K$-fold) distributions observed with the new bands lead to the mass assignment. Configurations for the two bands were determined through analysis of the rotational alignments of the structures and a comparison of the experimental $B(M 1) / B(E 2)$ ratios with theory. Tentative spin assignments are proposed for the $\pi h_{11 / 2} \nu i_{13 / 2}$ band, based on energy level systematics for other known sequences in neighboring odd-odd rhenium nuclei, as well as on systematics seen for the signature inversion feature that is well known in this region. The spin assignment for the $\pi h_{11 / 2} \nu\left(h_{9 / 2} / f_{7 / 2}\right)$ structure provides additional validation of the proposed spins and configurations for isomers in the ${ }^{176} \mathrm{Au} \rightarrow{ }^{172} \mathrm{Ir} \rightarrow{ }^{168} \mathrm{Re} \alpha$-decay chain.

PACS numbers: 21.10.Re, 23.20.Lv, 27.70.+q

\section{INTRODUCTION}

The spectroscopy for odd-odd nuclei is typically difficult due to the coupling of an unpaired quasiproton with an unpaired quasineutron. In particular, assigning spins and parities can be challenging as there is often a high density of states at low energies which leads to significant mixing of these levels. A recent $\alpha$-decay study of the oddodd nucleus ${ }^{176} \mathrm{Au}[1]$ observed two isomeric states that $\alpha$ decay into levels of ${ }^{172} \mathrm{Ir}$, with subsequent decay into isomers of ${ }^{168}$ Re. One chain results from a high-spin isomer $\left(8^{+}, 9^{+}\right)$in ${ }^{176} \mathrm{Au}$, while the other is from a low-spin one

\footnotetext{
*Present address: Department of Physics, California Polytechnic State University, San Luis Obispo, California 93407, USA

${ }^{\dagger}$ Present address: Physics Division, Argonne National Laboratory, Argonne, Illinois 60439, USA

¥Present address: Marshall Space Flight Center, Huntsville, AL 35812, USA

$\S$ Present address: U.S. Army Research Laboratory, Adelphi, Maryland 20783, USA

IPresent address: Physics Department, Millsaps College, Jackson, MS 39210, USA

** Present address: Department of Nuclear Physics, R.S.P.E, Australian National University, Canberra, A.C.T. 2601, Australia

$\dagger^{\dagger}$ Present address: Physics Division, Oak Ridge National Laboratory, Oak Ridge, Tennessee 37831, USA

¥¥Present address: National Nuclear Data Center, Brookhaven National Laboratory, Upton, New York 11973, USA

$\S \S$ Present address: Nuclear Physics Division, Bhabha Atomic Research Centre, Mumbai 400 085, India
}

$\left(3^{-}, 4^{-}\right)$. The results of the present high-spin study of states in ${ }^{168} \mathrm{Re}$ aides in the spin (tentatively assigned $6^{+}$ in Ref. [1]) and configuration assignments of the isomers observed in the high-spin chain.

In addition, a recent publication of the first rotational structures in ${ }^{166} \mathrm{Re}[2]$, the lightest odd-odd rhenium nucleus with known excitations, reported two $\gamma$-ray decay sequences. Tentative spin assignments were made; however, these could not easily be based on systematics due to the absence of sequences in ${ }^{168}$ Re. In light of the new ${ }^{168}$ Re results presented in this work, a systematic study may assist in the spin assignment for the $\pi h_{11 / 2} \nu i_{13 / 2}$ structure in ${ }^{166}$ Re.

\section{EXPERIMENTAL DETAILS}

The reaction ${ }^{55} \mathrm{Mn}+{ }^{118} \mathrm{Sn}$ populated excited states of ${ }^{168} \operatorname{Re}$ in the $5 n$-evaporation channel. A beam energy of $260 \mathrm{MeV}$ was selected and the ${ }^{55} \mathrm{Mn}$ beam was provided by the ATLAS facility at Argonne National Laboratory. Two stacked ${ }^{118} \mathrm{Sn}$ targets, each of $0.6 \mathrm{mg} / \mathrm{cm}^{2}$ thickness, were bombarded by this beam and the Gammasphere spectrometer [3], consisting of 101 Comptonsuppressed Ge detectors, was used to detect the $\gamma$ rays. A total of $2.3 \times 10^{9}$ four-fold or greater coincidence events were recorded and sorted into a Blue database [4]. A Radware [5] coincidence hypercube was generated from this database in order to construct the new ${ }^{168}$ Re level scheme. As this was a particularly weak reaction chan- 


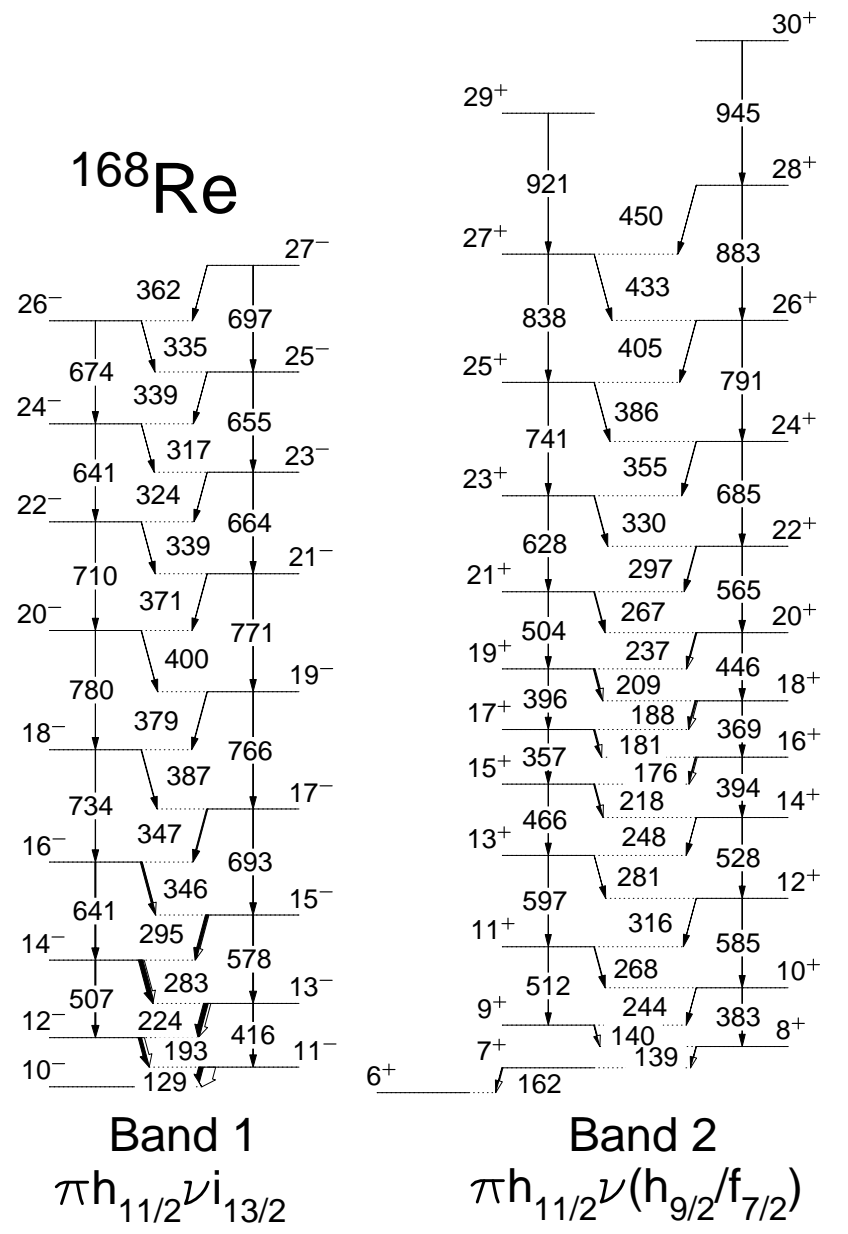

FIG. 1: Level scheme for ${ }^{168}$ Re from the present analysis. Since no direct measurements of spin or parity have been performed, all assignments are based on systematic arguments, as discussed in the text and, thus, must be considered tentative.

nel, the statistics were insufficient to perform a reliable angular correlation measurement. Therefore, the nature of the transitions ( $M 1$ or E2) is assumed based upon typical rotational behavior. The proposed level scheme for ${ }^{168} \mathrm{Re}$ is displayed in Fig. 1. The energies of both levels and $\gamma$ rays are given in Table $\mathrm{I}$. In addition, when a reliable branching ratio $(\lambda)$ could be measured (by gating above the state), this value is also provided in Table I.

TABLE I: Level and $\gamma$-ray energies, along with branching ratios $(\lambda)$ in ${ }^{168}$ Re. As the ground state is not known, and the two sequences are not linked, a different offset ( $X$ and $Y$ ) for the energy levels is postulated.

$$
\begin{array}{cccc}
I^{\pi[a]} E_{\text {level }}(\mathrm{keV}) & E_{\gamma}(\mathrm{keV})^{[b]} & \lambda^{[c]} \\
\hline \multicolumn{4}{c}{\text { Band 1: } \pi h_{11 / 2} \nu i_{13 / 2}} \\
10^{-} & \mathrm{X} & \\
11^{-} & \mathrm{X}+128.5 & 128.5 & \\
12^{-} & \mathrm{X}+321.1 & 192.6 & \\
13^{-} & \mathrm{X}+544.8 & 416.3 & 0.13(2)
\end{array}
$$

$$
\begin{aligned}
& 14^{-} \mathrm{X}+827.9 \quad 506.8 \\
& 15^{-} \mathrm{X}+1122.9-53.1 \\
& 15^{-} \mathrm{X}+1122.9 \quad 578.1 \\
& 16^{-} \mathrm{X}+1469.0 \quad 640.9 \\
& 17^{-} \mathrm{X}+1816.1 \\
& 347.1 \\
& 18^{-} \mathrm{X}+2203.2 \quad 734.1 \\
& 19^{-} \mathrm{X}+2582.5 \quad 766.3 \\
& 379.3 \\
& 20^{-} \quad \mathrm{X}+2982.7 \quad 779.6 \\
& 21^{-} \mathrm{X}+3353.6 \quad 771.2 \\
& 22^{-} \mathrm{X}+3693.0-370.9 \\
& 339.4 \\
& 23^{-} \mathrm{X}+4017.2 \quad 663.5 \\
& 24^{-} \mathrm{X}+4333.7 \quad 640.7 \\
& 25^{-} \mathrm{X}+4672.6 \quad 655.4 \\
& 338.9 \\
& 26^{-} \mathrm{X}+5007.3 \quad 673.6 \\
& 27^{-} \mathrm{X}+5369.5 \quad 696.9 \\
& \text { Band 2: } \pi h_{11 / 2} \nu\left(h_{9 / 2} / f_{7 / 2}\right) \\
& 7^{+} \mathrm{Y}+162.1 \quad 162.1 \\
& 8^{+} \quad \mathrm{Y}+301.2 \quad 139.1 \\
& 9^{+} \mathrm{Y}+441.1 \quad 139.9 \\
& 10^{+} \mathrm{Y}+685.3 \quad 383.0 \\
& 11^{+} \mathrm{Y}+953.5=24.2 \\
& 268.2 \\
& 12^{+} \mathrm{Y}+1269.9 \quad 584.6 \\
& 13^{+} \mathrm{Y}+1550.4 \quad 596.8 \quad 0.74(9) \\
& 14^{+} \mathrm{Y}+1798.1 \quad 528.3 \quad 0.70(11) \\
& 15^{+} \mathrm{Y}+2016.5 \quad 466.1 \quad 0.13(2) \\
& 16^{+} \mathrm{Y}+2192.5 \quad 394.3 \quad 0.12(2) \\
& 17^{+} \mathrm{Y}+2373.4 \quad 356.9 \quad 0.08(4) \\
& 18^{+} \mathrm{Y}+2561.0 \quad 368.6 \quad 0.14(2) \\
& 19^{+} \mathrm{Y}+2769.8 \quad 396.4 \quad 0.22(4) \\
& 20^{+} \mathrm{Y}+3006.6 \quad 445.5 \quad 0.20(5) \\
& 21^{+} \mathrm{Y}+3274.0 \quad 504.1 \quad 0.26(5) \\
& 22^{+} \mathrm{Y}+3571.2 \quad 564.5 \quad 0.32(8) \\
& 23^{+} \mathrm{Y}+3901.5 \quad 627.5 \quad 0.22(4) \\
& 24^{+} \mathrm{Y}+4256.5 \quad 685.4 \quad 0.50(12)
\end{aligned}
$$




\begin{tabular}{ccc}
$25^{+}$ & $\mathrm{Y}+4642.8$ & 355.0 \\
& & 741.3 \\
$26^{+}$ & $\mathrm{Y}+5047.9$ & 796.3 \\
& & 405.1 \\
$27^{+} \mathrm{Y}+5481.0$ & 838.2 \\
& & 433.1 \\
$28^{+} \mathrm{Y}+5931.0$ & 883.0 \\
& & 450.0 \\
$29^{+}$ & $\mathrm{Y}+6402.1$ & 921.1 \\
$30^{+} \mathrm{Y}+6876.3$ & 945.3 \\
\hline \hline
\end{tabular}

${ }^{a}$ Spin and parity of the depopulated state.

${ }^{b}$ Uncertainties in $\gamma$-ray energies are $0.2 \mathrm{keV}$ for most transitions.

${ }^{c}$ Branching ratio, $I_{\gamma}(E 2) / I_{\gamma}(M 1)$, where $I_{\gamma}$ is the intensity of the transition.

\section{LEVEL SCHEME}

Prior to this study, little was known concerning ${ }^{168}$ Re. Meissner et al. [6] performed an $e c / \beta^{+}$decay experiment to measure the halflife of the ground state, $t_{1 / 2}=4.4(1)$ $\mathrm{s}$, and estimated its spin/parity to be $I^{\pi}=\left(5^{+}, 6^{+}\right)$based on the feeding pattern toward the ${ }^{168} \mathrm{~W}$ daughter nucleus. An investigation of the $\alpha$ decay of ${ }^{172} \operatorname{Ir}[7]$ observed $\gamma$ ray transitions in coincidence with the $\alpha$ activity. The $\alpha$ decay from the high-spin isomer in ${ }^{172}$ Ir feeds an excited state in ${ }^{168}$ Re, which emits a $162-\mathrm{keV} \gamma$ transition, possibly directly to the ground state. Reference [8] confirmed the presence of this $\gamma$ ray, but tentatively assigned $I^{\pi}=\left(7^{+}\right)$to the lowest state. However, Ref. [1] recently suggested that this level is better associated with $I^{\pi}=\left(6^{+}\right)$. In addition, three other $\gamma$ rays $(90,123$, and $136 \mathrm{keV}$ ) were found in coincidence with the $\alpha$ decay of the low-spin ${ }^{172}$ Ir isomer [7].

Representative spectra for the two structures displayed in the level scheme of Fig. 1 are shown in Fig. 2. These sequences are associated with ${ }^{168}$ Re based upon the following reasoning: (1) both are clearly in coincidence with the characteristic $K_{\alpha}$ x ray of $61.1 \mathrm{keV}$, as seen in Figs. 2(a) and (b), which fixes the proton number $Z$ of the nucleus being investigated to 75 ; (2) structures resulting from evaporation channels that emit fewer particles will have a higher $K$ fold (sum of all detectors, Ge and BGO, that fired in an event) than those from channels with more particles. To first order, this results from the residual nucleus being left (following particle evaporation) at a higher excitation energy, so that more $\gamma$ rays will be emitted with the sequences associated with the $3 n$ channel in comparison to the $4 n$ or $5 n$ ones. In Gammasphere, the so-called $K$ fold is recorded for each event, and thus, this value can help distinguish which evaporation channel is responsible for a particular band. In Fig. 3, the $K$-fold distribution is provided for events that contained the 224- and 346-keV transitions from band 1 in Fig. 1. For comparison, the $K$-fold distributions for events associated with the 246 - and $365-\mathrm{keV}$ lines from the $\pi h_{11 / 2}$

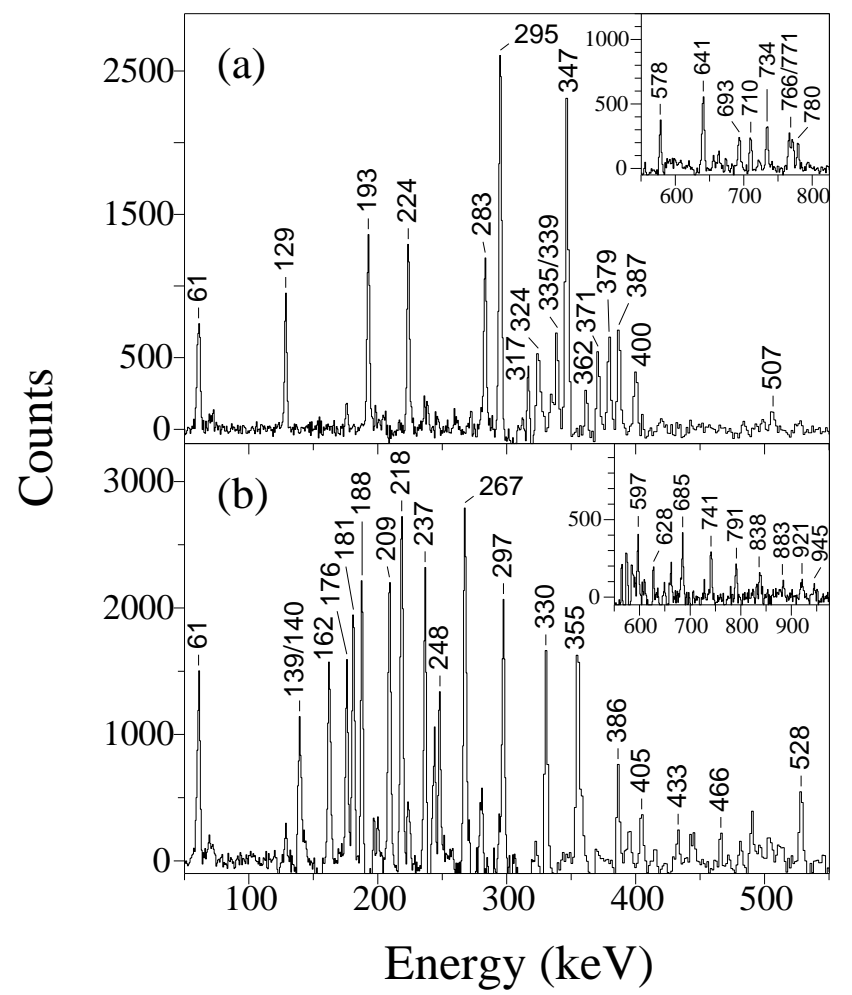

FIG. 2: (a) Coincidence spectrum of band 1 in ${ }^{168}$ Re resulting from the sum of all combinations of triple gates in the hypercube using the dipole transitions between the $12^{-}$and $20^{-}$ states, except for the $295-\mathrm{keV}$ transition. The inset displays the high-energy portion of the same spectrum. (b) Spectrum of band 2 which was produced in a similar manner as panel (a), where all combinations of triple gates using the dipole transitions between the $16^{+}$and $23^{+}$states were summed together. Once again, the inset displays the high-energy region of the same spectrum.

band in ${ }^{169} \operatorname{Re}$ (4n channel) [9] as well as those for events with the 189 - and $340-\mathrm{keV} \gamma$ rays from the $\pi h_{11 / 2} \nu i_{13 / 2}$ sequence in ${ }^{170} \operatorname{Re}$ ( $5 n$ channel) [9] are also displayed in Fig. 3. It should be noted that a normalization was applied such that the areas under the distribution curves are equal in order to better display the results. Clearly, a lower average $K$ value is observed for the events associated with band 1 (and a similar distribution is found for band 2) than those observed for ${ }^{169}$ Re and ${ }^{170}$ Re; therefore, bands 1 and 2 can be assigned to the $5 n$ reaction channel leading to ${ }^{168}$ Re. Nevertheless, a dedicated experiment utilizing a mass spectrometer would be desirable in order to firmly confirm this assignment.

The sequence labeled as band 1 in Fig. 1 has the largest intensity at lower spins $(<15)$, and the majority of this intensity is observed in the dipole transitions. Therefore, many of the latter transitions were used to produce the spectrum given in Fig. 2(a). The yield of the 295-keV line appears large since it was not included as one of the gating transitions (due to it having the same energy as a contaminant transition); however, the $347-\mathrm{keV}$ peak in 


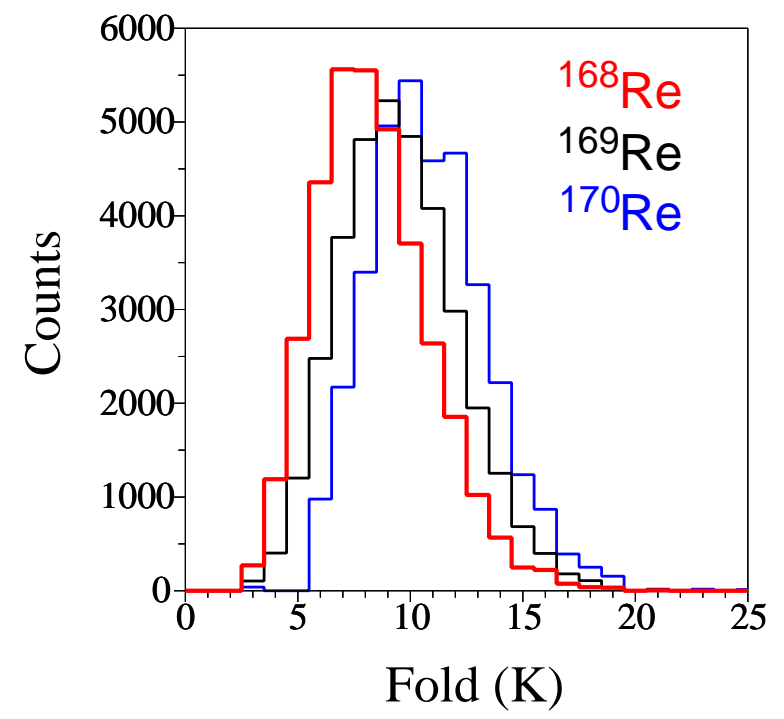

FIG. 3: (Color online) Normalized $K$-fold distributions (see text for definition) for events associated with strongly-coupled bands in ${ }^{169} \mathrm{Re}$ and ${ }^{170} \mathrm{Re}$, as well as the sequence labeled as band 1 in Fig. 1 . The lower $K$-fold distribution for band 1 indicates that it is associated with the $5 n$ reaction channel, leading to the assignment of the structure to ${ }^{168}$ Re.

the figure is also significantly larger than expected as it is a doublet. The spin and parity assignments for band 1 are based on the configuration assignment and a subsequent systematic analysis of similar structures in nearby odd-odd rhenium nuclei. Therefore, a discussion of this issue is given in Sec. IV.

Similar to band 1 , the dipole transitions carry the majority of the strength for band 2 . Once again, these transitions were used to produce the spectrum in Fig. 2(b). Two important items should be noted in this spectrum: (1) The presence of a $162-\mathrm{keV} \gamma$ ray further adds to the confidence of assigning band 2 to ${ }^{168}$ Re as it was also seen in the $\alpha$-decay works of Refs. [7, 8]. It is not clear at this time whether the $162-\mathrm{keV} \gamma$ is an inband transition, or if it decays to a separate structure; (2) careful inspection of Fig. 2(b) also suggests that there is a coincidence of band 2 with the 129-, 193-, 224-, and 295-keV lines from band 1. Unfortunately, no linking transitions could be identified; however, the observed coincidence between the two structures verifies that they belong to the same nucleus. Similar to band 1, the spins and parity of band 2 are based on the assigned configuration, as is discussed in Sec. IV.

\section{DISCUSSION}

In order to determine the configurations for bands 1 and 2 in ${ }^{168} \mathrm{Re}$, the alignments for both have been plotted in Fig. 4 versus the rotational frequency. Harris parameters [10] of $\mathcal{J}_{0}=17 \hbar^{2} / \mathrm{MeV}$ and $\mathcal{J}_{1}=50 \hbar^{4} / \mathrm{MeV}^{3}$ were chosen to subtract the angular momentum of the rotating

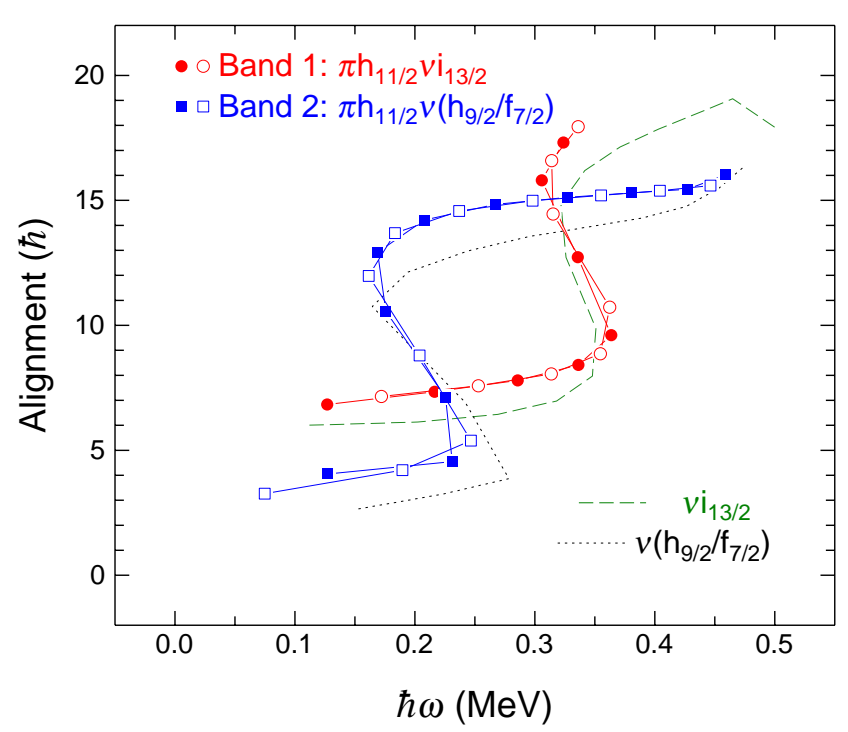

FIG. 4: (Color online) Alignments of bands 1 and 2 in ${ }^{168}$ Re. The Harris parameters used are discussed in the text. In addition, the $\nu i_{13 / 2}$ and $\nu\left(h_{9 / 2} / f_{7 / 2}\right)$ bands from ${ }^{167} \mathrm{~W}$ are plotted for reference.

core. The two sequences have different band crossings, where band 1 exhibits a backbend at $\hbar \omega_{c}=0.34 \mathrm{MeV}$, and band 2 at $\hbar \omega_{c}=0.20 \mathrm{MeV}$.

The alignments of the $\nu i_{13 / 2}$ and $\nu\left(h_{9 / 2} / f_{7 / 2}\right)$ bands in the isotone ${ }^{167} \mathrm{~W}_{93}$ [11] are also displayed in Fig. 4 as the dashed and dotted lines, respectively. Comparison of these sequences with bands 1 and 2 in ${ }^{168}$ Re allow for an assignment of the quasineutrons associated with the latter sequences. The $\nu i_{13 / 2}$ structure forms the yrast sequence of ${ }^{167} \mathrm{~W}$, and the well-known $B C$ crossing (corresponding to the second and third $i_{13 / 2}$ quasineutrons aligning) is observed at $\hbar \omega_{c}=0.34 \mathrm{MeV}$. In contrast, the mixed $\nu\left(h_{9 / 2} / f_{7 / 2}\right)$ sequence exhibits the $A B$ crossing (alignment of the lowest $i_{13 / 2}$ quasineutrons) at 0.22 $\mathrm{MeV}$. Therefore, the $i_{13 / 2}$ quasineutron must be associated with band 1 , while the $h_{9 / 2} / f_{7 / 2}$ quasineutron is involved in band 2. It should be noted that, even if the spins shown in Fig. 1 are incorrect, the crossing frequencies would not change drastically, and thus the arguments for the quasineutron assignments are nearly independent of the adopted spins values.

Inspection of the neighboring odd- $A$ rhenium nuclei, ${ }^{167} \operatorname{Re}[12]$ and ${ }^{169} \operatorname{Re}[9]$, indicates that the $\pi h_{11 / 2}$ state is energetically lower than any of the other quasiproton orbitals. Indeed, the $\pi h_{11 / 2}$ structure is populated significantly more intensely than any other. Therefore, this quasiproton can likely be associated with both bands in ${ }^{168}$ Re. In order to confirm this hypothesis, theoretical transition strength ratios were compared with experimental values, where the latter $B(M 1) / B(E 2)$ ratios were determined by: 


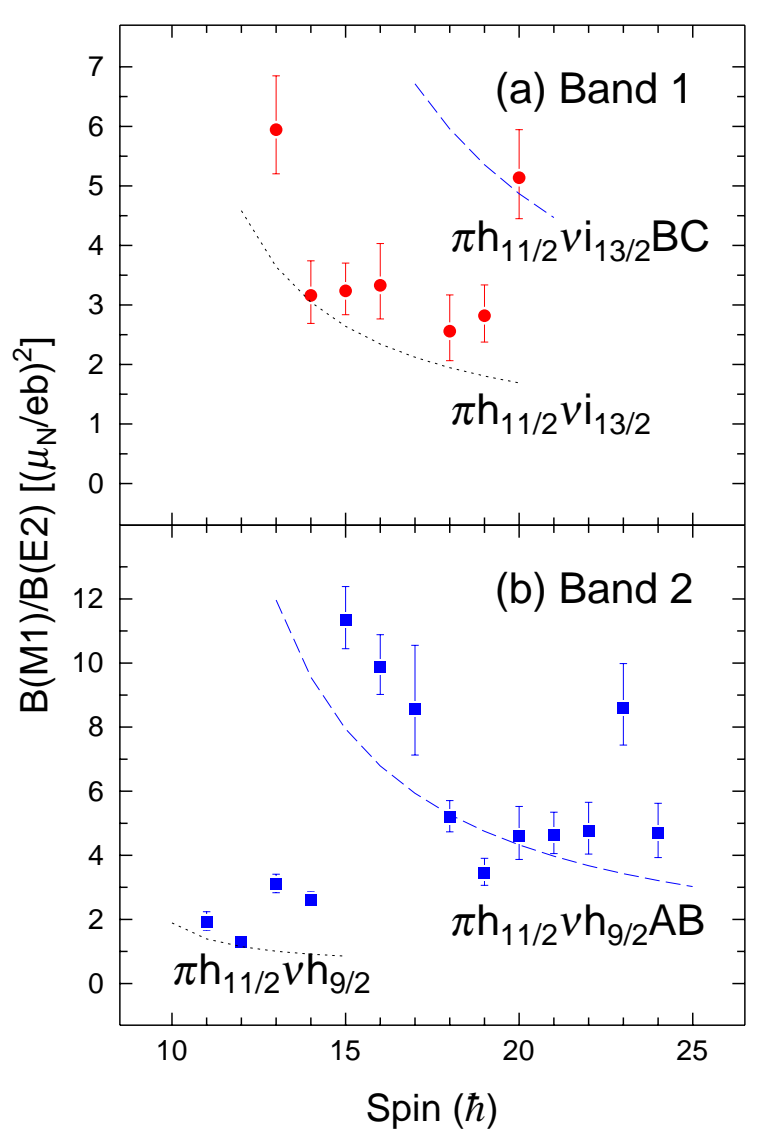

FIG. 5: (Color online) Experimental and theoretical $B(M 1) / B(E 2)$ ratios for the decay sequences in ${ }^{168} \mathrm{Re}$. Parameters for the theoretical calculations are discussed in the text and given in Table II.

$$
\frac{B(M 1: I \rightarrow I-1)}{B(E 2: I \rightarrow I-2)}=0.697 \frac{1}{\lambda\left(1+\delta^{2}\right)} \frac{E_{\gamma}^{5}(E 2)}{E_{\gamma}^{3}(M 1)}
$$

where $E_{\gamma}$ is given in $\mathrm{MeV}$, and the branching ratio $\lambda$ was determined from spectra with coincidence gates placed above the state of interest such that side feeding effects are eliminated. The branching ratios are given in Table I, and the mixing ratios $(\delta)$ were estimated from the rotational model that assumed pure $K$ values [13]. The corresponding $B(M 1) / B(E 2)$ ratios for bands 1 and 2 are plotted in Fig. 5. Theoretical ratios were calculated based on the geometrical approximation for $B(M 1)$ strengths [14] and the rotational form for the $B(E 2)$ reduced probabilities [13]. Parameters used in the calculation of the theoretical $B(M 1) / B(E 2)$ ratios are given in Table II, along with $g_{R}=Z / A=0.446$ and $Q_{0}=4.8 \mathrm{eb}$. These values are shown as dotted and dashed lines in Fig. 5, and good agreement can be seen for the selected configurations (both before and after the crossings) for bands 1 and 2 . Therefore, band 1 is assigned the $\pi h_{11 / 2} \nu i_{13 / 2}$ configuration, and band 2 the $\pi h_{11 / 2} \nu\left(h_{9 / 2} / f_{7 / 2}\right)$ one.
TABLE II: Parameters used in calculating the theoretical $B(M 1) / B(E 2)$ values shown in Fig. 5.

\begin{tabular}{lcc}
\hline \hline Configuration & $g_{\Omega}$ & $i_{x}(\hbar)$ \\
$\pi h_{11 / 2}$ & 1.30 & 1.4 \\
$\nu i_{13 / 2}$ & -0.3 & 6.0 \\
$\nu h_{9 / 2} / f_{7 / 2}$ & -0.32 & 2.0 \\
$\nu i_{13 / 2} A B$ & -0.3 & 11.0 \\
$\nu i_{13 / 2} B C$ & -0.3 & 8.0 \\
\hline \hline
\end{tabular}

With the configurations established, the spins of the structures can be addressed. Alignment is an additive property, therefore, this quantity can be used to help estimate the spins in the bands. In Fig. 4, one may observe that the $i_{13 / 2}$ quasineutron is associated with $\sim 6$ $\hbar$ of alignment at low frequencies. An initial alignment of $\sim 1.4 \hbar$ is associated with the $h_{11 / 2}$ quasiproton from the work on ${ }^{167,169}$ Re. By selecting a spin of $I^{\pi}=10^{-}$ for the lowest state in band 1, its alignment is approximately $1.3 \hbar$ above that of the $\nu i_{13 / 2}$ sequence from ${ }^{167} \mathrm{~W}$ at $\hbar \omega=0.2 \mathrm{MeV}$, which justifies the spin assignment shown in Fig. 1. In addition, a systematic inspection of the energy levels of the $\pi h_{11 / 2} \nu i_{13 / 2}$ bands in neighboring odd-odd rhenium nuclei can add further confirmation to this assignment. The low-spin levels for ${ }^{166} \operatorname{Re}[2],{ }^{168} \mathrm{Re}$, ${ }^{170} \operatorname{Re}[9],{ }^{172} \operatorname{Re}[15]$, and ${ }^{174} \operatorname{Re}[16]$ are plotted in Fig. 6 . Smooth trends are easily recognizable; however, it should be stated that the spins for ${ }^{166}$ Re were raised by $2 \hbar$ in comparison to those shown in Ref. [2]. Indeed, for a similar additivity of alignment to correctly describe this band in ${ }^{166} \mathrm{Re}$, the lowest state needs to be $I^{\pi}=10^{-}$, just as in ${ }^{168} \mathrm{Re}$.

An additional check for the suggested spin of the $\pi h_{11 / 2} \nu i_{13 / 2}$ band can be performed by inspecting the trends of signature splitting observed in neighboring oddodd nuclei. This configuration is well known for exhibiting signature inversion [17], where the expected unfavored signature (odd spins) lies lower in energy at low spins than the expected favored signature (even spins). Figure 7 displays the energy splitting between signatures, $S(I)$, of the $\pi h_{11 / 2} \nu i_{13 / 2}$ band for the $N=91$, 93, 95, 97, and 99 lutetium, tantalum, and rhenium nuclei. The quantity $S(I)$ is defined to be: $S(I)=$ $E(I)-E(I-1)-[E(I+1)-E(I)+E(I-1)-E(I-2)] / 2$ and the expected favored signature sequence (even spins) is shown as filled circles, while the expected unfavored one (odd spins) is displayed with open circles. In all cases, except for ${ }^{170} \mathrm{Lu}$, the odd-spin signature is lower in energy at lower spins. Various explanations for this effect have been discussed in many papers, such as Refs. [1721]; however, the purpose of Fig. 7 in the present work is to help provide justification for the assigned spin. Indeed, Liu et al. [22] used the systematics of signature splitting to help assign spins for the $\pi h_{11 / 2} \nu i_{13 / 2}$ band throughout the rare-earth region.

In Fig. 7, the marked spins are where the normal en- 


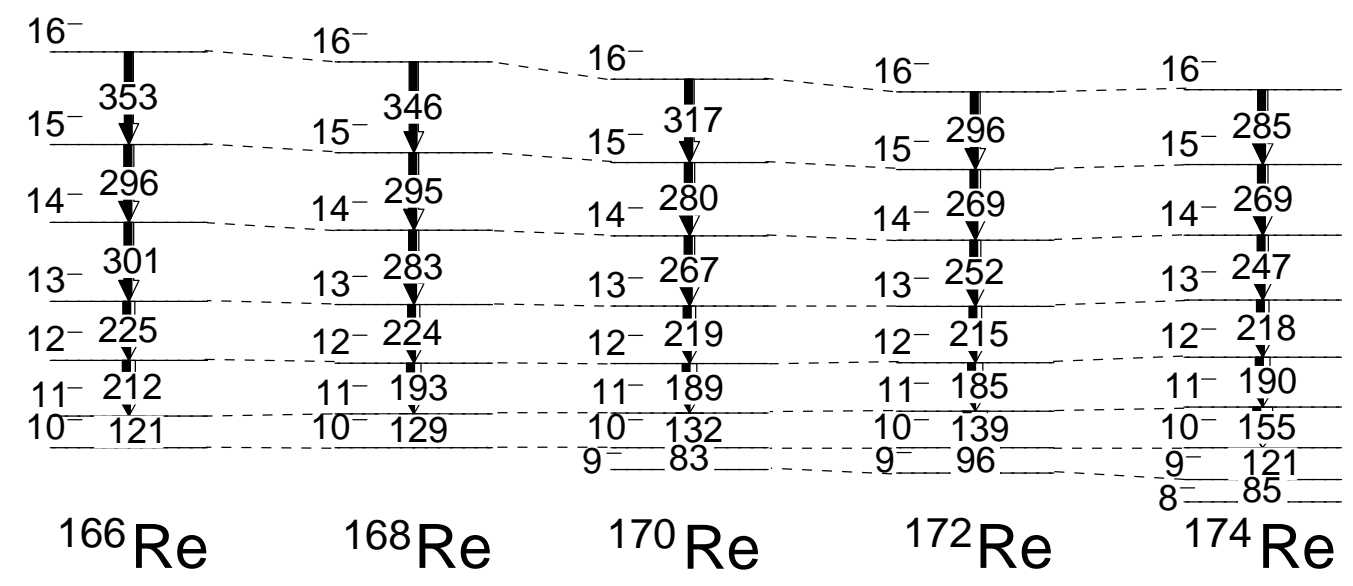

FIG. 6: Energy levels of the lowest $\pi h_{11 / 2} \nu i_{13 / 2}$ states in the lightest odd-odd rhenium nuclei.

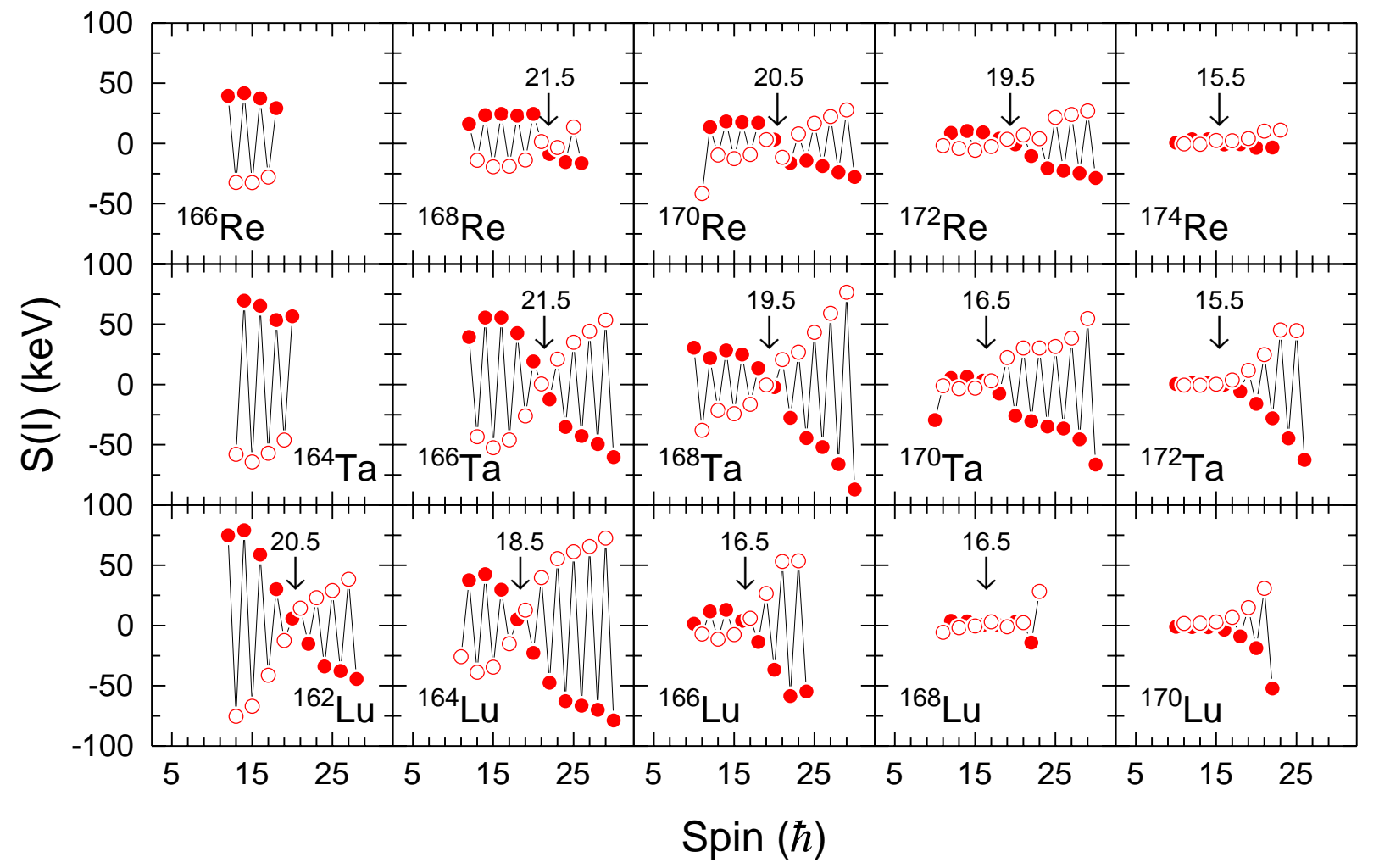

FIG. 7: (Color online) Signature splitting function, $S(I)$, as defined in the text, versus spin for $\pi h_{11 / 2} \nu i_{13 / 2}$ bands. Even spins (odd spins) are denoted with filled (open) circles. The reversion spins (as defined in text) are also labeled.

ergy ordering takes over (this is the so-called reversion spin) such that the even spin sequence lies lower in energy and signature inversion no longer applies. Clear trends can be observed with the reversion spin as this value becomes lower in an isotopic chain as the neutron number increases. In addition, the reversion spin increases systematically in an isotonic chain as the proton number increases. Using the proposed spin for the $\pi h_{11 / 2} \nu i_{13 / 2}$ band in ${ }^{168}$ Re (see Fig. 1) produces a signature inversion effect similar to that seen in neighboring odd-odd nuclei, and its reversion spin follows the systematics in both the isotopic and isotonic chains. Therefore, the spin assignment of band 1 appears to be in good agreement with the other $\pi h_{11 / 2} \nu i_{13 / 2}$ sequences in this region, herewith strengthening the proposed assignments.

Systematics are not readily available for band 2 since the $\pi h_{11 / 2} \nu\left(h_{9 / 2} / f_{7 / 2}\right)$ configuration is not observed widely in this region. However, one can derive a good estimate for the spins of band 2 using the additivity of alignment in a manner similar to that for band 1 . In Fig. 4, the alignment of the $\nu\left(h_{9 / 2} / f_{7 / 2}\right)$ sequence is shown, and using the assigned spins for band 2 given 
in Fig. 1, a similar $1.3 \hbar$ difference is found between these structures at $0.2 \mathrm{MeV}$. This is consistent with the $h_{11 / 2}$ quasiproton contributing $\sim 1.4 \hbar$ of alignment to the $\pi h_{11 / 2} \nu\left(h_{9 / 2} / f_{7 / 2}\right)$ configuration; therefore, the $I^{\pi}=7^{+}$ spin/parity assignment for the lowest spin state of band 2 appears to be appropriate.

In addition, the recent $\alpha$-decay work of Andreyev et al. [1] observed a single decay from the high-spin $7^{+}$isomer in ${ }^{172}$ Ir to an excited state in ${ }^{168}$ Re, which then emits a $162.1-\mathrm{keV} \gamma$ ray to a proposed $6^{+}$high-spin isomer. Since a 162.1-keV transition was observed in coincidence with band 2 from the present data, it is likely that this $\gamma$ ray corresponds to the one in the $\alpha$-decay study. Andreyev et al. [1] tentatively assigned the state at 162 $\mathrm{keV}$ as $7^{+}$, based upon the $\pi h_{11 / 2} \nu\left(h_{9 / 2} / f_{7 / 2}\right)$ configuration. By observing the rotational band based on this state in the present work and concluding that this level is likely to have $I^{\pi}=7^{+}$as well, one further justifies this spin/parity assignment. Consequently, this provides supporting evidence for the configuration assignments suggested in Ref. [1] for the ${ }^{176} \mathrm{Au},{ }^{172} \mathrm{Ir}$, and ${ }^{168}$ Re high-spin isomers. Indeed, these studies are a good example of how decay and high-spin experiments are complementary to each other.

\section{SUMMARY}

Two rotational structures have been assigned to the odd-odd nucleus ${ }^{168}$ Re. Configurations for both se- quences were proposed through the use of the observed alignments and $B(M 1) / B(E 2)$ transition strength ratios. Arguments for the spins of these bands are based on a variety of energy systematics, consistency checks, and the additive property of alignments. These spin assignments suggest a possible change for the spin given to the $\pi h_{11 / 2} \nu i_{13 / 2}$ sequence in ${ }^{166} \mathrm{Re}$ and add validation to the recent spin assignments for the $\alpha$-decaying isomers in the ${ }^{176} \mathrm{Au} \rightarrow{ }^{172} \mathrm{Ir} \rightarrow{ }^{168}$ Re chain.

\section{ACKNOWLEDGMENTS}

The authors thank the ANL operations staff at Gammasphere and gratefully acknowledge the efforts of J. P. Greene for target preparation. We thank D. C. Radford and H. Q. Jin for their software support. This work is funded by the National Science Foundation under Grant Nos. PHY-1203100 (USNA), PHY-0754674 (FSU), and PHY10-68192 (ND), as well as by the U.S. Department of Energy, Office of Nuclear Physics, under Contract Nos. DE-AC02-06CH11357 (ANL), DEFG02-94ER40848 (UML), DE-FG02-96ER40983 (UT), DE-FG02-95ER40939 (MSU) and DE-FG02-94ER40834 (UMCP). This research used resources of Argonne National Laboratory's ATLAS facility, which is a DOE Office of Science User Facility.
[1] A. N. Andreyev et al., Phys. Rev. C 90, 044312 (2014).

[2] H. J. Li et al., Phys. Rev. C 92, 014310 (2015).

[3] R. V. F. Janssens and F. S. Stephens, Nucl. Phys. News 6, 9 (1996).

[4] M. Cromaz et al., Nucl. Inst. Meth. A 462, 519 (2001).

[5] D. C. Radford, Nucl. Inst. Meth. A 361, 297 (1995).

[6] F. Meissner, H. Salewski, W.-D. Schmidt-Ott, U. BoschWicke, and R. Michaelsen, Z. Phys. A 343, 283 (1992).

[7] W.-D. Schmidt-Ott, H. Salewski, F. Meissner, U. BoschWicke, P. Koschel, V. Kunze, and R. Michaelsen, Nucl. Phys. A545, 646 (1992).

[8] J. TM. Goon, Thesis, University of Tennessee (2004).

[9] D. J. Hartley et al., Phys. Rev. C 87, 024315 (2013).

[10] S. M. Harris, Phys. Rev. 138B, 509 (1965).

[11] K. Theine et al., Nucl. Phys. A548, 71 (1992).

[12] D. T. Joss et al., Phys. Rev. C 68, 014303 (2003).

[13] A. Bohr and B. R. Mottelson, Nuclear Structure, Vol. II
(Benjamin, New York), 1975.

[14] F. Dönau, Nucl. Phys. A471, 469 (1987).

[15] D. J. Hartley et al., Phys. Rev. C 90, 017301 (2014).

[16] S. Guo et al., Phys. Rev. C 86, 014323 (2012).

[17] R. Bengtsson, H. Frisk, F. R. May, and J. A. Pinston, Nucl. Phys. A415, 189 (1984).

[18] P. B. Semmes and I. Ragnarsson, Proc. Inter. Conf. Nuclear Structure of the Nineties, Oak Ridge, Tennessee, Vo. 1, p. 62 (1990).

[19] K. Hara and Y. Sun, Nucl. Phys. A531, 221 (1991).

[20] N. Tajima, Nucl. Phys. A572, 365 (1994).

[21] F. R. Xu, W. Satula, and R. Wyss, Nucl. Phys. A669, 119 (2000).

[22] Yunzuo Liu, Yingjun Ma, Hongting Yang, and Shangui Zhou, Phys. Rev. C 52, 2514 (1995). 